\title{
Scan time and patient dose for thoracic imaging in neonates and small children using axial volumetric 320-detector row CT compared to helical 64-, 32-, and 16- detector row CT acquisitions
}

\author{
Lucia J. M. Kroft • Joost J. H. Roelofs • Jacob Geleijns
}

Received: 11 May 2009/Revised: 7 August 2009/Accepted: 19 September 2009 / Published online: 8 December 2009

(C) The Author(s) 2009. This article is published with open access at Springerlink.com

\begin{abstract}
Background Recently a 320-detector-row CT (MDCT) scanner has become available that allows axial volumetric scanning of a $16-\mathrm{cm}$-long range $(50 \mathrm{~cm}$ field of view) in a single 0.35 -s rotation. For imaging neonates and small children, volume scanning is potentially of great advantage as the entire scan range can be acquired in $0.35 \mathrm{~s}$, which can reduce motion artefacts and may reduce the need for sedation in clinical CT imaging. Also, because there is no over-ranging associated with axial volumetric scanning, this may reduce patient radiation dose.

Objective To evaluate, by means of a phantom study, scan time and patient dose for thoracic imaging in neonates and small children by using axial cone-beam and helical fanbeam MDCT acquisitions.

Materials and methods Paediatric imaging protocols were assessed for a 320-MDCT volumetric scanner (Aquilion ONE, Toshiba, Otawara, Japan). The 320-MDCT scanner allows for cone-beam acquisitions with coverage up to $160 \mathrm{~mm}$, but it also allows for helical fan-beam acquisitions in 64-, 32-, or 16-MDCT modes. The acquisition configurations that were evaluated were $320 \times 0.5 \mathrm{~mm}, 240 \times$ $0.5 \mathrm{~mm}$, and $160 \times 0.5 \mathrm{~mm}$ for axial volumetric scanning, and $64 \times 0.5 \mathrm{~mm}, 32 \times 0.5 \mathrm{~mm}$, and $16 \times 0.5 \mathrm{~mm}$ for helical scanning. Dose assessment was performed for clinically relevant paediatric angiographic or chest/mediastinum acquisition protocols with tube voltages of 80 or $100 \mathrm{kVp}$ and tube currents between 40 and $80 \mathrm{~mA}$.

Results Scan time was $0.35 \mathrm{~s}$ for 320-MDCT acquisitions, scan times varied between $1.9 \mathrm{~s}$ and $8.3 \mathrm{~s}$ for helical
\end{abstract}

L. J. M. Kroft $(\varangle) \cdot$ J. J. H. Roelofs · J. Geleijns

Department of Radiology, Leiden University Medical Center,

Albinusdreef 2, C2-S,

Leiden 2333 ZA, The Netherlands

e-mail: L.J.M.Kroft@lumc.nl acquisitions. Dose savings varying between $18 \%$ and $40 \%$ were achieved with axial volumetric scanning as compared to helical scanning (for 320- versus 64-MDCT at $160 \mathrm{~mm}$ and $80 \mathrm{kVp}$, and for 320 - versus 16-MDCT at $80 \mathrm{~mm}$ and $100 \mathrm{kVp}$, respectively). Statistically significant reduction in radiation dose was found for axial 320-MDCT volumetric scanning compared to helical 64-, 32-, and 16-MDCT scanning.

Conclusion Axial thoracic CT of neonates and small children with volumetric 320-MDCT can be performed between 5 and 24 times faster compared to helical scanning and can save patient dose.

Keywords Multidetector CT · Dose · Children

\section{Introduction}

Helical scanning is currently the standard acquisition method for thoracic CT. In recent years developments in CT technology have provided increasing temporal and better spatial resolution. Scan times are much shorter and slice thickness much thinner with increasing rotation speed and increasing number of active detector-rows, from 4 and 16 detector rows to 64-detector CT (MDCT) scanners.

A drawback of increasing nominal beam width in helical scanning is the increase in z-over-ranging; the effect is most prominent when acquiring a small scan range. Over-ranging is the phenomenon that occurs in helical acquisitions where the actual exposed range exceeds the imaged range. Corresponding extra rotations are required for proper interpolations during the reconstruction process for images at the borders of the imaged range $[1,2]$. The effect of overranging on patient dose becomes larger for helical scanners with more detector rows and wider coverage. 
Recently, a 320-MDCT scanner has become available that allows axial volumetric scanning of a $16 \mathrm{~cm}$ long range $(50 \mathrm{~cm}$ field of view $(\mathrm{FOV}))$ in a single $0.35 \mathrm{~s}$ rotation. For imaging neonates and small children, volume scanning is potentially of great advantage as the entire scan range can be acquired in $0.35 \mathrm{~s}$, which can reduce motion artefacts and may reduce the need for sedation in clinical $\mathrm{CT}$ imaging. Also, because there is no over-ranging associated with axial volumetric scanning, this may reduce patient radiation dose.

Radiation dose to children is of particular concern since they are more susceptible to radiation hazards than adults, due to rapidly proliferating tissues and, therefore, being more sensitive to tumour induction and longer postexposure life expectancy (increasing the probability that an induced tumour will manifest clinically) [3].

Based on the technical features of the 320-MDCT, it is hypothesized that axial volumetric cone-beam instead of helical fan-beam acquisition would potentially be advantageous for imaging neonates and small children. Therefore, a study was designed to assess the effect of scan time and dose savings of axial volumetric CT compared to helical CT. The purpose of this study was to evaluate, by means of a phantom study, scan time and patient dose for thoracic imaging in neonates and small children by using axial cone beam and helical fan beam MDCT acquisitions.

\section{Materials and methods}

\section{Data acquisition}

Paediatric imaging protocols were assessed for a 320MDCT volumetric scanner (Aquilion ONE, Toshiba, Otawara, Japan). The 320-MDCT scanner allows for cone-beam acquisitions with coverage up to $160 \mathrm{~mm}$, but also allows for helical fan-beam acquisitions in 64-, 32-, or 16-MDCT modes. The acquisition configurations that were evaluated were $320 \times 0.5 \mathrm{~mm}, 240 \times 0.5 \mathrm{~mm}$, and $160 \times$ $0.5 \mathrm{~mm}$ for axial volumetric scanning and $64 \times 0.5 \mathrm{~mm}, 32 \times$ $0.5 \mathrm{~mm}$, and $16 \times 0.5 \mathrm{~mm}$ for helical scanning. Dose assessment was performed for clinically relevant paediatric angiographic or chest/mediastinum acquisition protocols with tube voltages of 80 or $100 \mathrm{kVp}$ and tube currents between 40 and $80 \mathrm{~mA}$. The imaging protocol uses higher tube voltage and higher tube current for children of older age and greater weight, and thus compensates for different attenuation in neonates and small children. Imaging parameters used were: FOV $240 \mathrm{~mm}$; rotation time $0.35 \mathrm{~s}$ for axial volumetric and helical scanning; recommended pitch factor (PF) for helical acquisitions 0.828 for 64MDCT, 0.844 for 32-MDCT, and 0.938 for 16-MDCT.

For MDCT chest imaging from the lung bases to the apices, it was assumed that for children up to 5 years, 16- $\mathrm{cm}$ scan range would be sufficient, whereas for older (taller) children $16-\mathrm{cm}$ scan range might be too short $[4,5]$. Craniocaudal scan ranges of $80 \mathrm{~mm}, 120 \mathrm{~mm}$, and $160 \mathrm{~mm}$, representing relevant scan ranges for thoracic imaging in children, were used. Other scan ranges can be applied in clinical practice to adapt the scan range to the individual.

Tube current can be varied in discrete steps of $10 \mathrm{~mA}$, an appropriate tube current was selected to yield similar effective mAs for the helical acquisition configurations compared to the mAs in the axial cone-beam acquisition. Effective mAs incorporates a correction for the PF, and is defined to facilitate comparison of $\mathrm{mAs}$ for axial acquisitions with helical acquisitions at different PFs. By selecting similar effective $\mathrm{mAs}$ for the different acquisitions, we achieved similar radiation output of the CT scanner for the different axial and helical acquisitions. The resulting tube current for thoracic $\mathrm{CT}$ imaging in small children is shown in Table 1. Noise measurements in a cylindrical water phantom (diameter $21 \mathrm{~cm}$, length $24 \mathrm{~cm}$; Toshiba, Otawara, Japan) confirmed that the selected tube current provides image noise (standard deviation [SD] of Hounsfield units) in a comparable range for the different acquisition configurations (320-, 64-, 32-, and 16-MDCT) at either $80 \mathrm{kVp}$ or $100 \mathrm{kVp}$ (Table 1) [6, 7]. The use of the same phantom for noise measurements is appropriate for comparing noise within the well-defined age and weight groups; however, it does not allow for comparisons between the different paediatric patient groups.

For the acquisition conditions described above, the weighted CT dose index $\left(\mathrm{CTDI}_{\mathrm{w}}\right)$ was derived from measurements with a 300-mm-long CT pencil ionization chamber (CT-110, Applied Engineering Inc., Japan) in an extended 350-mm-long CT dose head phantom (diameter $160 \mathrm{~mm}$ ) [8]. Dose length product (DLP) was calculated taking into account the applied PF (only helical scans) and the exposed range. Total scan time was recorded for all acquisition protocols. The effect of z-over-ranging for the helical 64-, 32-, and 16-MDCT acquisitions was taken into account in both DLP and total scan time. DLP can be used as a practical and reliable measure for the total amount of radiation dose received by the child during the scan [9-12].

\section{Data analysis}

The total scan time for each acquisition, and differences between the acquisition techniques are provided as numeric data and as a percentage. Differences in DLP radiation dose between the MDCT scan modes as well as the effect of $\mathrm{z}$ over-ranging are expressed as numeric data as mean $\pm \mathrm{SD}$ and as a percentage. The paired $t$-test was used to determine differences in radiation dose measures between the 320 and 64-, 32-, and 16-MDCT scan modes. $P$-values $<0.05$ were considered statistically significant. 
Table 1 Required tube current $(\mathrm{mA})$ for achieving comparable effective $\mathrm{mA}$ (and image noise) in different helical acquisition configurations compared to the axial 320-MDCT cone beam acquisition (rotation time $0.35 \mathrm{~s}$ ). Variable tube current settings for comparable image quality in the helical acquisitions yielded comparable noise-levels (SD of Hounsfield units) for thoracic helical scanning protocols compared to axial 320-MDCT acquisitions. Helical scanning was performed with recommended PFs

\begin{tabular}{|c|c|c|c|c|c|}
\hline Acquisition mode & Tube voltage $(\mathrm{kV})$ & $\begin{array}{l}\text { 320-MDCT } \\
\text { Axial }\end{array}$ & $\begin{array}{l}\text { 64-MDCT } \\
\text { Helical } \\
(\mathrm{PF}=0.828)\end{array}$ & $\begin{array}{l}\text { 32-MDCT } \\
\text { Helical } \\
(\mathrm{PF}=0.844)\end{array}$ & $\begin{array}{l}\text { 16-MDCT } \\
\text { Helical } \\
(\mathrm{PF}=0.938)\end{array}$ \\
\hline \multicolumn{2}{|l|}{ Age and weight category } & \multicolumn{4}{|c|}{ Tube current; effective tube current ( ) in mA CTDIvol in mGy; noise level [ ] } \\
\hline \multirow[t]{2}{*}{ Neonate or $<4 \mathrm{~kg}$} & 80 & $60(60)$ & $50(60)$ & $50(59)$ & $60(64)$ \\
\hline & & $1.5[55]$ & $1.4[69]$ & $1.7[70]$ & $2.1[64]$ \\
\hline \multirow[t]{2}{*}{$<1$ year or $4-<10 \mathrm{~kg}$} & 80 & $80(80)$ & $60(72)$ & $70(83)$ & $70(75)$ \\
\hline & & $2.0[47]$ & $1.7[60]$ & $2.3[54]$ & $2.4[57]$ \\
\hline \multirow[t]{2}{*}{$1-2.5$ years or $10-<20 \mathrm{~kg}$} & 100 & $50(50)$ & $40(48)$ & $40(47)$ & $50(53)$ \\
\hline & & $2.4[39]$ & $2.2[43]$ & $2.4[44]$ & $3.3[39]$ \\
\hline \multirow[t]{2}{*}{$\leq 5$ years or $20-<30 \mathrm{~kg}$} & 100 & $80(80)$ & $60(72)$ & $70(83)$ & $70(75)$ \\
\hline & & $3.8[30]$ & $3.2[33]$ & $4.2[31]$ & $4.6[32]$ \\
\hline
\end{tabular}

\section{Results}

Acquisition time

Total scan time for axial volumetric scanning was $0.35 \mathrm{~s}$ (one single rotation) for all 320-MDCT protocols. With helical scanning, acquisition times varied between 1.9 and $8.3 \mathrm{~s}$, corresponding respectively to the fastest $80-\mathrm{mm}$ coverage helical 64-MDCT and to the slowest $160-\mathrm{mm}$ coverage helical 16-MDCT protocol. With axial 320MDCT, the acquisition time was reduced by $82-93 \%$ (a factor of 5-13 times faster) for the 80-mm scan range, 85$95 \%$ (a factor of 7-18 times faster) for the $120-\mathrm{mm}$ scan range, and $88-96 \%$ (a factor of 8-24 times faster) for the $160-\mathrm{mm}$ scan range, as compared to $64-$ and 16-MDCT helical scan modes, respectively. Values of the 32-MDCT scan modes are between those obtained with the 64- and 16-MDCT scan modes (Fig. 1).

\section{Dose saving}

DLP for axial 320-, and helical 64-, 32-, and 16-MDCT scan modes are given in Table 2. Dose savings varying between $18 \%$ and $40 \%$ were achieved with axial volumetric scanning as compared to helical scanning (for 320- versus 64-MDCT at $160 \mathrm{~mm}$ and $80 \mathrm{kVp}$, and for 320- versus 16MDCT at $80 \mathrm{~mm}$ and $100 \mathrm{kVp}$, respectively). Statistically significant reduction in radiation dose was found for axial 320-MDCT volumetric scanning compared to helical 64-, $32-$, and 16-MDCT scanning. Mean DLP values were $28.8 \pm$ $12.7 \mathrm{mGy} \cdot \mathrm{cm}$ for 320 -MDCT, $38.3 \pm 14.7 \mathrm{mGy} \cdot \mathrm{cm}$ for $64-$ MDCT $(P<0.001), 40.2 \pm 17.5 \mathrm{mGy} \cdot \mathrm{cm}$ for 32-MDCT $(P<$ $0.001)$, and $43.0 \pm 17.6 \mathrm{mGy} \cdot \mathrm{cm}$ for 16-MDCT $(P<0.001)$. Corresponding mean percentage dose saving for 320MDCT was $25 \%$ compared to $64-\mathrm{MDCT}, 28 \%$ compared to $32-\mathrm{MDCT}$, and $33 \%$ compared to $16-\mathrm{MDCT}$. The smaller the scan range, the more dose reduction was achieved by the application of axial 320-MDCT. Figure 2 shows the graphic representations of the mean DLP values for the four scan protocols. Also note that the $\mathrm{CTDI}_{\mathrm{vol}}$ increases (within categories of age and weight) when less active detector rows are selected; this is a well-known effect and results from the so-called penumbra effect that is more prominent at smaller beam widths (Table 1).

\section{Z-over-ranging}

Table 3 shows the actual exposed range and the z-overranging (as a percentage of the imaged range) for each scan

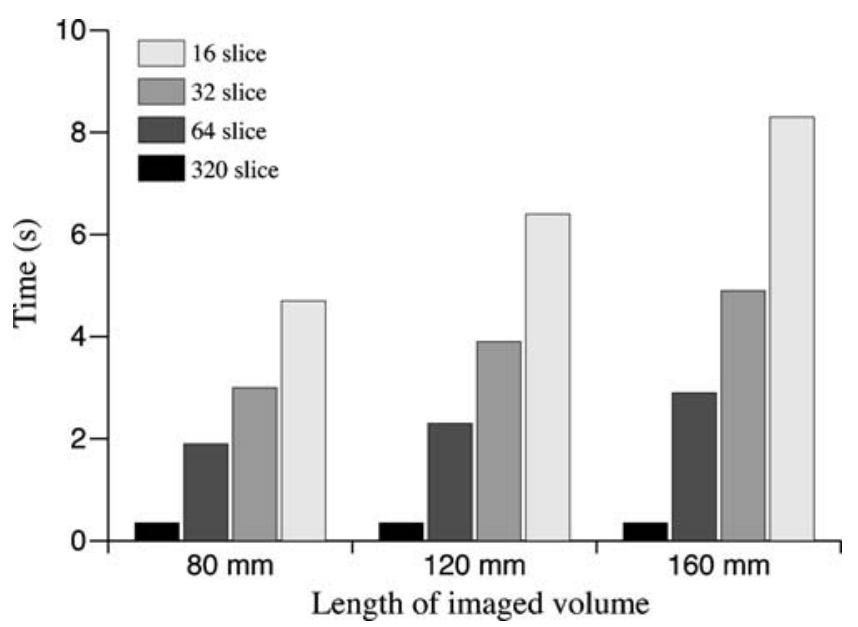

Fig. 1 Acquisition time for thoracic CT imaging protocols of varying scan length for neonates and small children performed with 320-, 64-, $32-$, and $16-\mathrm{MDCT}$ scanners. Note the $(0.35 \mathrm{~s})$ scan time that remains constant for scan ranges up to $16 \mathrm{~cm}$ with the volumetric 320 -MDCT acquisition as compared to longer and increasing scan times for the 64-, 32-, and 16-MDCT helical scanners 
Table 2 Calculated patient dose expressed as DLP $(\mathrm{mGy} \cdot \mathrm{cm})$ for 320-, 64-, 32-, and 16-MDCT scan modes (rotation time $0.35 \mathrm{~s})$. Tube current (mA) reference values for 320-MDCT adopted by calibration for 64-, 32-, and 16-MDCT are shown in Table 1

\begin{tabular}{|c|c|c|c|c|c|}
\hline $\begin{array}{l}\text { Acquisition mode } \\
\mathrm{kV} / \mathrm{mA} \text { reference value patient category }\end{array}$ & Imaged range $(\mathrm{mm})$ & $\begin{array}{l}\text { 320-MDCT } \\
\text { Axial } \\
\text { Dose length }\end{array}$ & $\begin{array}{l}\text { 64-MDCT } \\
\text { Helical } \\
\text { ct (mGy.cm) }\end{array}$ & $\begin{array}{l}\text { 32-MDCT } \\
\text { Helical }\end{array}$ & $\begin{array}{l}\text { 16-MDCT } \\
\text { Helical }\end{array}$ \\
\hline $80 \mathrm{kV} / 60 \mathrm{~mA}$ & 80 & 12.9 & 20.7 & 19.2 & 21.1 \\
\hline \multirow[t]{2}{*}{ Neonate or $<4 \mathrm{~kg}$} & 120 & 18.4 & 25.1 & 24.9 & 28.7 \\
\hline & 160 & 23.4 & 31.6 & 31.3 & 37.2 \\
\hline $80 \mathrm{kV} / 80 \mathrm{~mA}$ & 80 & 17.2 & 24.9 & 26.8 & 24.6 \\
\hline \multirow[t]{2}{*}{$<1$ year or $4-<10 \mathrm{~kg}$} & 120 & 24.5 & 30.1 & 34.9 & 33.5 \\
\hline & 160 & 31.3 & 38.0 & 43.8 & 43.4 \\
\hline $100 \mathrm{kV} / 50 \mathrm{~mA}$ & 80 & 19.7 & 31.0 & 27.9 & 33.1 \\
\hline \multirow[t]{2}{*}{$1-2.5$ years or $10-<20 \mathrm{~kg}$} & 120 & 28.2 & 37.5 & 36.3 & 45.1 \\
\hline & 160 & 36.0 & 47.3 & 45.6 & 58.5 \\
\hline $100 \mathrm{kV} / 80 \mathrm{~mA}$ & 80 & 31.6 & 46.5 & 48.8 & 46.4 \\
\hline \multirow[t]{2}{*}{$\leq 5$ years or $20-<30 \mathrm{~kg}$} & 120 & 45.2 & 56.3 & 63.5 & 63.1 \\
\hline & 160 & 57.5 & 71.0 & 79.7 & 81.9 \\
\hline
\end{tabular}

mode and each scan range. The proportion of z-overranging varied between $11 \%$ and $80 \%$ for helical scanning (16- and 64-MDCT, respectively). Z-over-ranging increased at larger nominal beam width and at smaller scan range. No z-over-ranging occurs at axial volumetric CT scanning (320-MDCT) based on phantom measures performed with the same scan parameters.

Figure 3 shows the images of a neonate scanned with fast volumetric $320-\mathrm{MDCT}$. The 4 -week-old $3.5-\mathrm{kg}$ boy

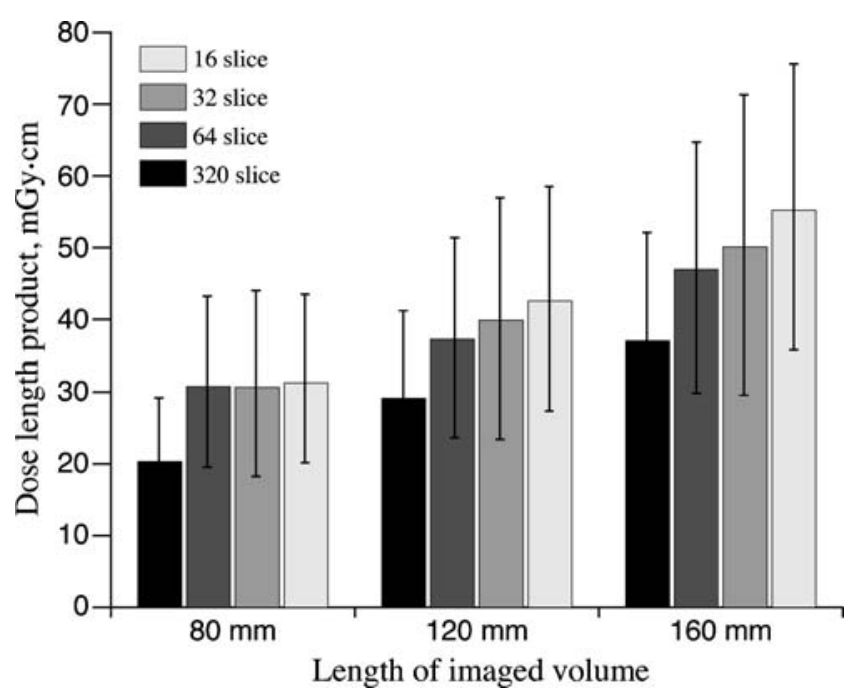

Fig. 2 Mean radiation dose expressed as DLP for thoracic CT imaging protocols of varying scan length for neonates and small children performed with 320-, 64-, 32-, and 16-MDCT scanners. Note that the radiation doses are lowest for the volumetric 320-MDCT acquisitions as compared to the doses required with 64-, 32-, and 16MDCT helical scanning. Mean DLP values were statistically significant lower for 320-MDCT than for helical 64-, 32-, and 16MDCT; all $P$-values $<0.001$ had unexplained serious hypoxic events 2 weeks after arterial switch operation. Right ventricular outflow tract / pulmonary artery compression or obstruction by postoperative haematoma was suspected, but the postoperative area could not be visualized well by echocardiography. Sternotomy was planned for diagnosis and CT imaging was requested. After CT evaluation, sternotomy for diagnosis was no longer required. The DLP was $16.6 \mathrm{mGy} \cdot \mathrm{cm}$, based on phantom measurements performed with same scan parameters. Estimated effective patient radiation dose was $16.6 \mathrm{mGy} \cdot \mathrm{cm} \times 0.039 \mathrm{mSv} / \mathrm{mGy} \cdot \mathrm{cm}$ (i.e. age-specific effective dose for chest imaging) $=0.65 \mathrm{mSv}$ [11].

\section{Discussion}

This phantom study has evaluated to what extent acquisition time and patient dose savings can be achieved for thoracic imaging in neonates and small children by using axial volumetric MDCT instead of helical MDCT acquisition. Acquisition protocols were used that yield similar image quality for the different MDCT scanning modes. All measurements were performed on the same scanner that allowed axial as well as helical acquisitions, thereby providing standard conditions where differences found could be ascribed to differences in scanning techniques only. The main findings were that for standard thoracic CT scans of neonates and small children a factor of 5-24 times faster scanning was achieved by volumetric MDCT as compared to helical MDCT. Time saving was most obvious for the largest scan range of $160 \mathrm{~mm}$. At the same time, with volumetric MDCT, dose savings varying between $18 \%$ and $40 \%$ were achieved a compared to helical MDCT. The 
Table 3 Imaged range and exposed range including $\mathrm{z}$ overscanning (excluding penumbra)

\begin{tabular}{lcccc}
\hline \multicolumn{5}{c}{ Exposed range (mm) } \\
\hline Imaged range (mm) & $320-$ MDCT & $64-\mathrm{MDCT}$ & $32-\mathrm{MDCT}$ & $16-\mathrm{MDCT}$ \\
80 & 80 & $143(80 \%)$ & $115(45 \%)$ & $100(26 \%)$ \\
120 & 120 & $174(45 \%)$ & $150(25 \%)$ & $137(14 \%)$ \\
160 & 160 & $219(37 \%)$ & $189(18 \%)$ & $177(11 \%)$ \\
\hline
\end{tabular}

smaller the craniocaudal scan range, and the greater the number of detectors rows used for helical scanning (i.e. 64and 16-MDCT), the greater the percent dose reduction achieved with axial 320-MDCT.

Potential reduction in patient radiation dose by volumetric scanning using a prototype 256-MDCT compared to 16MDCT has been reported previously for standard $120 \mathrm{kVp}$ applications in adults [13]. In that study, dose saving achieved for chest imaging with a scan range of $38 \mathrm{~cm}$ was $28 \%$ with axial volumetric 256-MDCT compared to helical 16-MDCT. The dose reduction that can be achieved with axial volumetric scanning compared to helical scanning can be explained by larger beam width of the volumetric MDCT scanner. The actual beam width in a MDCT scanner is the nominal beam width (the product of slice thickness and number of slices) plus a margin that covers the penumbra [13]. The relative contribution of the penumbra becomes less prominent for beams with a larger nominal beam width. Another important aspect that explains patient
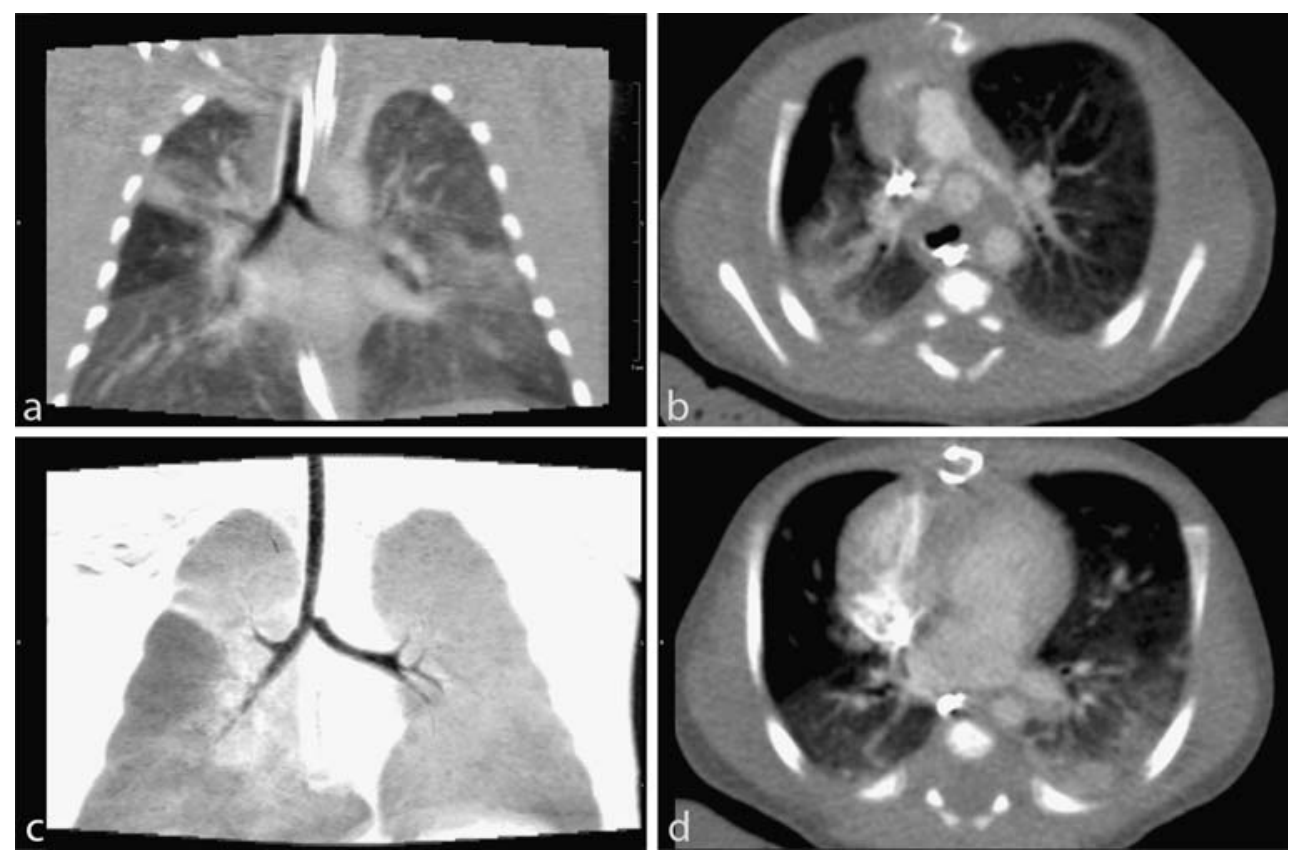

Fig. 3 A 4-week-old 3.5-kg boy. Imaging was performed using $6 \mathrm{ml}$ of contrast medium and $3 \mathrm{ml}$ saline by manual injection with $20 \mathrm{~s}$ scan delay. Volumetric imaging of an $8-\mathrm{cm}$ chest range was acquired using a $320-\mathrm{MDCT}$ scanner. Scan parameters were: tube voltage $80 \mathrm{kVp}$, tube current $60 \mathrm{~mA}$, rotation time $0.35 \mathrm{~s}$ that resulted in $21 \mathrm{mAs}$. A ventilation tube was in situ (a) and the central airways were found dose reduction with axial volumetric scanning as,compared to helical scanning is $\mathrm{z}$ - over-ranging (or overscanning) at helical acquisitions, which is absent in axial acquisitions. In helical scan mode, the reconstruction algorithm requires additional raw data on both sides of the planned scan range; therefore in helical mode the exposed range exceeds the imaged range. These extra helical rotations at the boundaries outside the planned area contribute to radiation dose, but not to image formation [1]. In axial volumetric acquisitions the exposed range corresponds exactly to the imaged range. Therefore, z-over-ranging is not applicable to volumetric scanning, resulting in a more effective use of radiation for image formation with volumetric acquisition [2].

Although the advantage of volumetric scanning over helical scanning due to lack of z-over-ranging has been well recognized, volumetric scanning has not been applied in neonates or small children for relative large scan ranges (such as chest imaging) with 64-, 32-, or 16-MDCT. While open (c). Substantial atelectasis was observed (a, b, d). There were small pulmonary arteries without obstruction after arterial switch operation, no right ventricular compression and/or substantial postoperative haematoma (b, d). After CT evaluation, sternotomy for diagnosis was no longer necessary 
knowledge of the effect of z-over-ranging was obtained with 16-MDCT scanners, performing volumetric acquisitions using these scanners would not be practical for imaging these specific patient groups $[1,2]$. This is because only small volumes with a maximum range of $32 \mathrm{~mm}$ are, for example, obtained per axial rotation for a $64 \mathrm{MDCT}$ acquisition (by using a Toshiba Aquilion 64-MDCT). Multiple axial rotations would be necessary to image a reasonable scan range. However, multiple rotation acquisitions would hamper "smooth" volume acquisition due to patient motion between these rotations, which is likely to cause step artefacts within the volume. With the 320MDCT a maximum $160 \mathrm{~mm}$ volume is acquired per rotation (simultaneously and without step artefacts) that is well within clinical scan ranges for thoracic imaging in neonates and small children.

Dual-source CT (DSCT) is another recent development and is also used for paediatric radiology [14]. This technology uses two X-ray tubes and two detectors and DSCT particularly improves temporal resolution. Coverage of the volume of interest with DSCT is achieved by a helical acquisition, and requires several rotations. DSCT optimizes the temporal resolution of the scan, but the scan time is longer compared to (axial) volumetric 320-MDCT. Scan time of the DSCT thoracic acquisition described is estimated at the order of magnitude of the 64-slice acquisitions presented in Fig. 1 [14].

In this study the advantage of axial volumetric scanning has been investigated for chest applications only. However, axial volumetric scanning may be applied to numerous clinical situations including abdominal imaging in children and in adults and also for brain, neck, liver, shoulder, hip and knee imaging. In addition, the 320-MDCT scanner allows prospective-gated imaging of the entire heart and coronary arteries within a single rotation, and ventricular functional imaging within a single heart beat.

The reduction in acquisition time and radiation dose that can be achieved with the volumetric MDCT scanner is of particular advantage when imaging neonates and small children. Scan times varying between $1.9 \mathrm{~s}$ and $8.3 \mathrm{~s}$ as measured here for state-of-the-art helical 64-, 32- and 16MDCT acquisitions with fast rotation time of $0.35 \mathrm{~s}$, are more prone to image artefacts than the single 0.35 -s acquisition time required with volumetric 320-MDCT scanning. The sub-second acquisition time with volumetric scanning may obviate the need for sedation in these children.

Neonates and small children are more susceptible to radiation hazards compared to adults. The sensitivity for stochastic radiation effects (carcinogenesis and genetic effects) of newborns and small children is a factor 10-3 times higher than in adults $[3,15,16]$. Their relatively high susceptibility to radiation and long post-exposure life expectancy requires dedicated paediatric scan protocols [3].
Lowering tube voltage $(\mathrm{kVp})$ and tube current $(\mathrm{mA})$ can be used as effective measures for reducing radiation dose to children $[6,11,17-19]$. It has been shown that for a given tube current, using a tube voltage of $100 \mathrm{kVp}$ instead of the traditionally used $120 \mathrm{kVp}$ results in a $34 \%$ effective dose reduction in children. With $80 \mathrm{kVp}$ instead of using $120 \mathrm{kVp}, 68 \%$ dose reduction can be achieved [18]. Comparable results were found in another study, where noise level was used as a measure of image quality [6].

At a given tube voltage, by using lower tube current settings in paediatric examinations, effective doses in paediatric chest $\mathrm{CT}$ examinations can be lowered by a factor of two or greater as compared to adult examinations [19]. Automatic tube current control, which adapts the acquisition to patient size and shape, effectively contributes to the optimization of CT acquisitions. DLP values are much lower in children than in adults due to the smaller scan lengths, and with proper adaptation of the tube settings, the effective doses in children can be much lower than in adults. Effective patient dose for a standard chest $\mathrm{CT}$ is estimated approximately $1.7 \mathrm{mSv}$ in newborns and approximately $5.4-7 \mathrm{mSv}$ in a normal-size adult $[17,20]$. Our findings of low DLP in small children, regardless of scan mode, are in line with these estimates.

There are some study limitations that should be addressed. Technology for beam collimation in CT evolves steadily and contributes to the reduction of radiation exposure. Some recent CT scanners from various manufacturers are equipped with a dynamic collimator. The dynamic collimator optimizes the longitudinal dose profile at the start and end of the helical scanned range; it reduces over-ranging, and thus reduces radiation exposure in helical CT scanning [21]. Collimation of axial volumetric CT scanners is being improved by tighter collimation, leading to reduced overbeaming, and thus reduced radiation exposure. The effect of a dynamic collimator in helical scans and the effect of tighter collimation in axial cone beam scans were not investigated in this study. Also, single-rotation axial MDCT imaging results in cone-shaped image borders at the cranial and caudal sides (Fig. 3). Therefore, the diagnostic area available at the peripheral borders of the scanned range is somewhat reduced, although this effect is of minor importance in small objects.

Dose calculations were based on measurement with a single $16-\mathrm{cm}$ dose phantom. Although this might not be fully representative of all patient variations in body shape that may occur in clinical practice, it provides for reasonable dose estimations in children. For craniocaudal scan ranges exceeding $16 \mathrm{~cm}$ an extra gantry rotation would be needed; together with table shift this would result in an approximately $1.0-2.0 \mathrm{~s}$ extra scan time. However, in our experience, scan ranges exceeding $16 \mathrm{~cm}$ are not required for chest imaging in neonates and small children and full 
chest imaging is performed in $0.35 \mathrm{~s}$ rotation. We used DLP to quantify patient dose $[4,10]$. Calculation of effective dose in children would allow for calculating risk related parameters and comparison with other radiation-based imaging modalities $[17,22,23]$.

\section{Conclusion}

Axial thoracic CT of neonates and small children with volumetric 320-MDCT can be performed between 5 and 24 times faster compared to helical scanning and can save patient dose.

Open Access This article is distributed under the terms of the Creative Commons Attribution Noncommercial License which permits any noncommercial use, distribution, and reproduction in any medium, provided the original author(s) and source are credited.

\section{References}

1. Van der Molen A, Geleijns J (2007) Overranging in multisection CT: quantification and relative contribution to dose-comparison of four 16-section CT scanners. Radiology 242:208-216

2. Tzedakis A, Damilakis J, Perisinakis K et al (2007) Influence of z overscanning on normalized effective doses calculated for pediatric patients undergoing multidetector $\mathrm{CT}$ examinations. Med Phys 34:1163-1175

3. Brenner D, Elliston C, Hall E et al (2001) Estimated risks of radiation-induced fatal cancer from pediatric CT. Am J Roentgenol 176:289-296

4. Khursheed A, Hillier MC, Shrimpton PC et al (2002) Influence of patient age on normalized effective doses calculated for CT examinations. Br J Radiol 75:819-830

5. Shrimpton PC, Hillier MC, Lewis MA et al (2006) National survey of doses from CT in the UK: 2003. Br J Radiol 79:968-980

6. Cody DD, Moxley DM, Krugh KT et al (2004) Strategies for formulating appropriate MDCT techniques when imaging the chest, abdomen, and pelvis in pediatric patients. Am J Roentgenol 182:849-859

7. Huda W, Scalzetti EM, Roskopf M (2000) Effective doses to patients undergoing thoracic computed tomography examinations. Med Phys 27:838-844

8. Geleijns J, Salvadó Artells M, de Bruin PW et al (2009) Computed tomography dose assessment for a $160 \mathrm{~mm}$ wide, 320 detector row, cone beam CT scanner. Phys Med Biol 54:3141-3159
9. International Electrotechnical Commission (2003) Particular requirements for the safety of x-ray equipment for computed tomography In: International standard of IEC 60601-2-44 2nd edition Amendment1: medical electrical equipment, Part 2-4 Geneva, Switzerland: International Electrotechnical Commission

10. International Commission on Radiological Protection (2007) Managing Patient Dose in Multi-Detector Computed Tomography (MDCT) IN: ICRP Publication 102: Volume 102. Annals of the ICRP Volume 37/1. International Commission on Radiological Protection series

11. Huda W, Ogden MK, Khorasani MR (2008) Converting dose length product to effective dose at CT. Radiology 248:9551003

12. Thomas KE, Wang B (2008) Age-specific effective doses for pediatric MSCT examinations at a large children's hospital using DLP conversion coefficients: a simple estimation method. Pediatr Radiol 38:645-656

13. Mori S, Endo M, Nishizawa K et al (2006) Comparison of patient doses in 256-slice CT and 16-slice CT scanners. Br J Radiol 79:56-61

14. Ben Saad M, Rohnean A, Sigal-Cinqualbre A et al (2009) Evaluation of image quality and radiation dose of thoracic and coronary dual-source CT in 110 infants with congenital heart disease. Pediatr Radiol 39:668-676

15. International Commission on Radiological Protection (2008) Recommendations of the ICRP In: ICRP Publication 103. Annals of the ICRP Volume 37/2-4. International Commission on Radiological Protection series Elsevier

16. Theocharopoulos N, Damilakis J, Perisinakis K et al (2006) Estimation of effective doses to adult and pediatric patients from multislice computed tomography: a method based on energy imparted. Med Phys 33:3846-3856

17. Huda W (2007) Radiation doses and risks in chest computed tomography examinations. Proc Am Thorac Soc 4:316-320

18. Lee C, Lee C, Staton RJ et al (2007) Organ and effective doses in pediatric patients undergoing helical multislice computed tomography examination. Med Phys 34:1858-1874

19. Fujii K, Aoyama T, Koyama S et al (2007) Comparative evaluation of organ and effective doses for paediatric patients with those for adults in chest and abdominal CT examinations. $\mathrm{Br}$ J Radiol 80:657-667

20. Mettler FA Jr, Huda W, Yoshizumi TT et al (2008) Effective doses in radiology and diagnostic nuclear medicine: a catalog. Radiology 248:254-263

21. Deak PD, Langner O, Lell M et al (2009) Effects of adaptive section collimation on patient radiation dose in multisection spiral CT. Radiology 252:140-147

22. Huda W, Atherton JV, Ware DE et al (1997) An approach for the estimation of effective radiation dose at $\mathrm{CT}$ in pediatric patients. Radiology 203:417-422

23. Fearon T, Vucich J (1987) Normalized pediatric organabsorbed doses from CT examinations. Am J Roentgenol 148:171-174 